\section{EFFECT OF VANCOMYCIN INFUSION ON CARDIAC FUNCTION IN PATIENTS SCHEDULED FOR CARDIAC OPERATION}

From the Department of Anesthesiology, University of Michigan Hospitals, Ann Arbor, Mich.

Received for publication Jan. 20, 1994.

Accepted for publication April 7, 1994.

Address for reprints: Jack Rosenberg, MD, Department of Anesthesiology, University of Michigan Hospitals, Ann Arbor, MI 48109. 0048

Copyright (0 1995 by Mosby-Year Book, Inc. $0022-5223 / 95 \$ 3.00+0 \quad \mathbf{1 2 / 1 / 5 6 8 1 5}$
Patients scheduled for cardiac operation often receive vancomycin before the operation to decrease postoperative staphylococcal wound infections. In animal studies, vancomycin depressed cardiac function approximately $15 \%$. Because of the potentially serious consequences of myocardial depression in patients undergoing cardiac operation, we examined the effect of vancomycin infusion on cardiac hemodynamics in patients scheduled for cardiac operation. Patients who were scheduled for cardiac operation and vancomycin prophylaxis were enrolled in our study. After baseline cardiac output, mean arterial pressure, central venous pressure, and pulmonary capillary wedge pressure were measured, $1 \mathrm{gm}$ of vancomycin $\mathrm{HCl}$ was infused over 1 hour. Cardiac output, mean arterial pressure, central venous pressure, and pulmonary capillary wedge pressure were measured at $15,30,60,90$, and 120 minutes after the start of the infusion. In the 46 patients that completed the study, no significant change was observed in cardiac output or systemic vascular resistance at any time when compared with baseline. Mean arterial pressure increased significantly $(p=0.03)$ between baseline $(90.8 \pm 2.4$ standard error of mean) and 90 minutes $(94.1 \pm 2.4$ standard error of mean). One patient had a transient $30 \%$ fall in mean arterial pressure and systemic vascular resistance with facial flushing during the infusion. In conclusion, we found that vancomycin infusion over 1 hour in patients before cardiac operation is safe and not associated with cardiac depression. (J THORAC CARDIOvaSC SuRg 1995;109:561-4)

J. M. Rosenberg, MD, J. A. Wahr, MD, and K. A. Smith, RN, BSN, Ann Arbor, Mich.
V ancomycin is used in the preoperative period to decrease postoperative staphylococcal wound infections. ${ }^{1,2}$ However, vancomycin depressed cardiac function approximately $15 \%$ in clinically relevant doses in open chest dogs and in Langendorff cat heart preparations. ${ }^{3}$ Peak effects were noted 1 hour after administration. When patients received vancomycin, $1 \mathrm{gm}$ was infused over 30 minutes before cardiac operation; hypotension occurred in $25 \%$ of patients $(>20 \%$ decrease in mean arterial pressure [MAP]) and was severe ( $>50 \%$ decrease) in $8 \%$ of patients compared with baseline. ${ }^{4}$ Because of the potentially serious consequences of cardiac depression in patients undergoing cardiac operation, we examined the effect of vancomycin on cardiac hemodynamics in patients scheduled for cardiac operation.

\section{Methods}

After obtaining approval from the institutional review board and informed consent, patients receiving vancomycin before cardiac operation were enrolled in our study. After baseline cardiac output (CO), MAP, central venous pressure (CVP), and pulmonary capillary wedge pressure (PCWP) were measured, $1 \mathrm{gm}$ of vancomycin $\mathrm{HCl}$ (Lilly Research Laboratories, Indianapolis, Ind.) diluted in $50 \mathrm{cc}$ of $5 \%$ dextrose in water, was administered over 1 hour by means of an infusion pump (Baxter Healthcare Corp., Santa Ana, Calif.). The vancomycin infusion and all experimental measurements were completed during the patients' 2-hour resting period in the preoperative holding room, during which no other medications were infused. Hemodynamic measurements were repeated at $15,30,60$, 90 , and 120 minutes after the start of the infusion. Cardiac outputs were the average of three end expiration measurements and measured with a 7.5F Swan-Ganz thermodilution catheter (Baxter Healthcare) connected to an -Abbott cardiac output computer (Abbott Laboratories, N. Chicago, Ill.). Typical preoperative sedation was administered and included midazolam or lorazepam and fentanyl which was given at least 30 minutes before the experiment. The patients also received their usual cardiac medications. No antihistamines or steroids were given. Data were analyzed by analysis of variance for repeated measures with the Scheffe $\mathrm{F}$ test. Individual differences were analyzed with unpaired $t$ tests. Reliability measures were determined with the methods of Vonesh and Schork. ${ }^{5}$

\section{Results}

Of the 52 patients enrolled in the study, eight patients were removed because they underwent operation before data collection was complete. Of the 46 patients who completed the study, 34 under- 


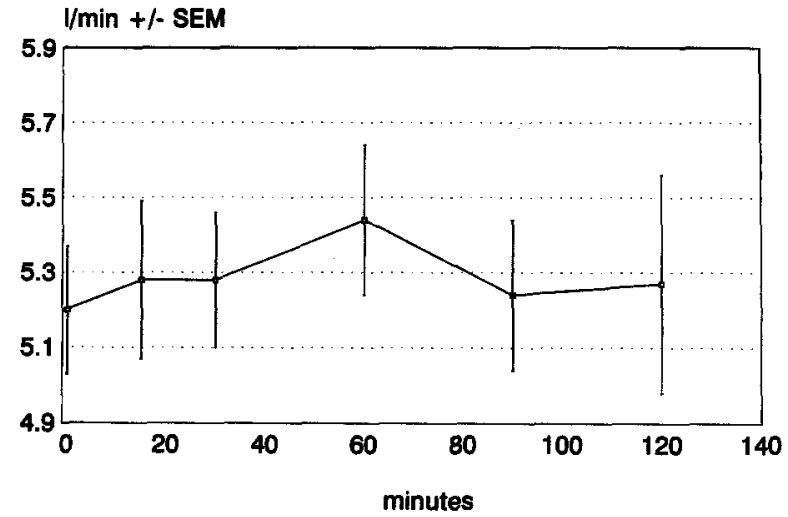

Fig. 1. Cardiac output ( $\mathrm{CO}$ ) during vancomycin infusion ( 0 to 60 minutes) and 60 minutes after. Values are means \pm SEM; no differences had a level of significance $\leq 0.05$.

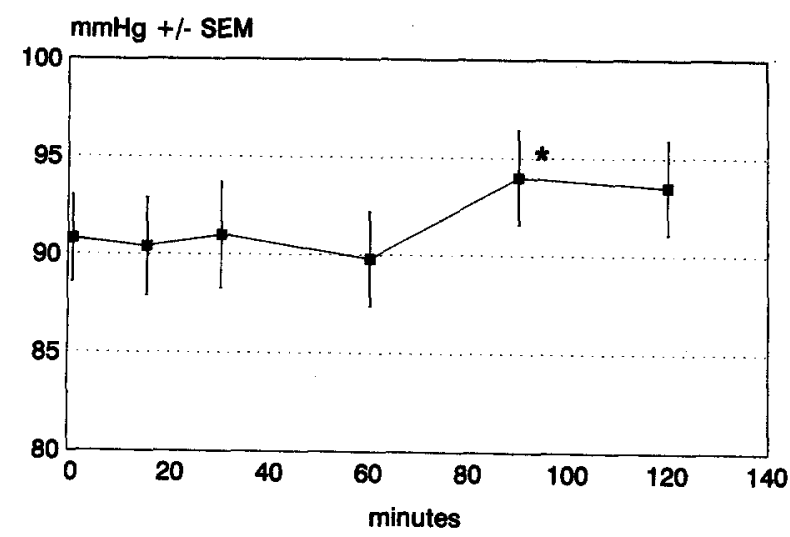

Fig. 2. MAP during vancomycin infusion (0 to $60 \mathrm{~min}-$ utes) and 60 minutes after. Values are means \pm SEM. *Level of significance for difference from other values $\leq 0.05$.

went coronary artery bypass grafting, eight had aortic valve replacement, and four had mitral valve replacement. No significant $(p<0.05)$ difference at baseline was found between the patients undergoing valve operations compared with those undergoing coronary artery bypass grafting in CO, MAP, or SVR.

Vancomycin infusion had no effect on $\mathrm{CO}$, as shown in Fig. 1. This result did not change when the patients undergoing coronary artery bypass grafting or valve operations were compared separately. The changes seen in MAP are shown in Fig. $2(p=0.03)$. A small increase in MAP between baseline (90.8 \pm 2.24 standard error of mean [SEM]) and 90 minutes (94.1 $\pm 2.4 \mathrm{SEM}$ ) was found with the use of $t$ tests. Vancomycin infusion had no effect on SVR, as shown in Fig. 3. One patient had a transient 30\% fall

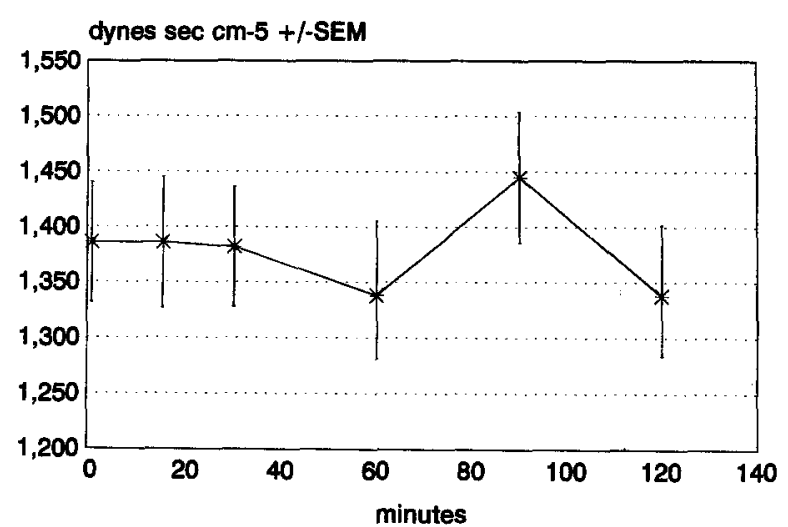

Fig. 3. SVR during vancomycin infusion (0 to $60 \mathrm{~min}$ utes) and 60 minutes after. Values are means \pm SEM; no differences had a level of significance $\leq 0.05$.

in MAP and SVR with facial flushing during the infusion.

The probability that a $15 \%$ decrease in cardiac output is related to vancomycin infusion that was not detected by this study is less than 1 in 1000 (power $\geq 0.999$ ).

\section{Discussion}

Our results show that 1 gm of vancomycin can be safely infused over 1 hour and that it is not associated with a decrease in $\mathrm{CO}$ in patients with known cardiac disease. These results are contrary to widespread concerns ${ }^{6-8}$ about vancomycin-induced cardiac depression in patients with poor cardiac function according to the 1972 animal studies of Cohen and associates. ${ }^{3}$ Decreased $\mathrm{CO}$ has not been associated with vancomycin infusion in human beings except in case reports of rapid infusions with lethal outcomes. ${ }^{9,10}$

The cardiac effects of vancomycin in human beings have been examined by two groups of investigators. Romanelli and associates ${ }^{11}$ administered 1 gm of vancomycin over 30 minutes to 30 patients on arrival to the operating room, after induction, and every 12 hours after the operation and compared hemodynamic measurements with those of 28 patients receiving normal saline solution. Measurements were taken before induction, after induction, during the operation, and every 4 hours for 24 hours after the operation. They found no difference between the groups before and after induction. Increased $\mathrm{CO}$ and heart rate with a decreased SVR and MAP were found after the operation in the vancomycin group, which necessitated vasopressor administration. These results were consistent with 
Table I. Hemodynamic changes during vancomycin infusion (values \pm SEM)

\begin{tabular}{llccccc}
\hline & Baseline & $15 \mathrm{~min}$ & $30 \mathrm{~min}$ & $60 \mathrm{~min}$ & $90 \mathrm{~min}$ & $120 \mathrm{~min}$ \\
\hline CO & $5.2 \pm 0.17$ & $5.28 \pm 0.21$ & $5.28 \pm 0.18$ & $5.44 \pm 0.2$ & $5.24 \pm 0.2$ & $5.27 \pm 0.29$ \\
MAP & $90.8 \pm 2.2$ & $90.4 \pm 2.5$ & $91 \pm 2.7$ & $89.8 \pm 2.4$ & $94 \pm 2.4^{*}$ & $93.5 \pm 2.4$ \\
SVR & $1386 \pm 54$ & $1386 \pm 59$ & $1382 \pm 54$ & $1338 \pm 57$ & $1445 \pm 59$ & $1338 \pm 54$ \\
\hline
\end{tabular}

${ }^{*} p \leq 0.05$.

vasodilation. Their experiment, although it shows vasodilatory properties of vancomycin, does not adequately examine whether vancomycin is a cardiac depressant. Specifically, vancomycin was not administered by infusion pump, the time period of predicted greatest effect coincided with induction of anesthesia, and the number of subjects was not sufficient to detect a $20 \%$ difference in cardiac output as predicted by the data of Cohen and associates. ${ }^{3}$ Furthermore, the vasodilatory effects were noted only after several doses of vancomycin, which makes it unclear whether it was a vancomycin effect.

Stier and associates ${ }^{12}$ examined the hemodynamic effects of $1 \mathrm{gm}$ of vancomycin infused over 30 minutes given to 16 patients after their cardiac operation. No significant hemodynamic changes were found, and one patient had symptoms consistent with "red-man syndrome," but the number of patients was not sufficient to detect a $20 \%$ decrease in CO. Most (13 of 16) of the patients had already received vancomycin in the intraoperative period before experimental measurements.

The prevalence of hypotension in patients receiving vancomycin before cardiac operation was examined in the recent study by Valero, Gomar, and Fita. ${ }^{4}$. They found a $25 \%$ prevalence of hypotension when vancomycin was infused over 30 minutes after induction of anesthesia. CO was not reported. Other studies in patients without cardiac operation included less hypotension. ${ }^{13,14}$ We did not experience a similar reduction in MAP when the vancomycin was administered before the operation over a longer period (60 minutes), except in one patient who had a $30 \%$ decrease in MAP and SVR with no change in $\mathrm{CO}$. Although not addressed directly by this investigation, the difference in rates of hypotension can be explained as a disparity in the magnitude of the anaphylactoid response to vancomycin (altered SVR) and not a direct myocardial depression.

The prevalence of anaphylactoid reaction to vancomycin infusion is $0 \%$ to $90 \%$, depending on dose, infusion time, definition of reaction, and concomi- tant medications. Experimental studies show a doserelated increase in histamine release and that the severity of the symptoms is directly related to histamine level. Hypotension does not develop during rapid infusion of vancomycin when dogs are given $\mathrm{H} 1$ blockers. It is reasonable to conclude that hypotension is a severe response to histamine release in a spectrum of responses ranging from flushing to cardiovascular collapse. ${ }^{14}$

Differences in speed of administration alter peak serum levels. One gram of vancomycin infused over 60 minutes produced mean plasma concentrations of $63 \mathrm{mg} / \mathrm{L}$ at the completion of the infusion and 23 $\mathrm{mg} / \mathrm{L} 2$ hours after the infusion (Lilly Research Laboratories, unpublished data, 1984). Infusion of $500 \mathrm{mg}$ over 30 minutes produces mean serum levels of $49 \mathrm{mg} / \mathrm{L}$. Infusion of 1 gram over the same 30 minute interval would likely yield serum levels near $100 \mathrm{mg} / \mathrm{L}$. Increased serum vancomycin and thus increased serum histamine is probably responsible for the $25 \%$ prevalence of hypotension seen by Valero, Gomar, and Fita. ${ }^{4}$ Whether anesthetic agents contributed to the hypotension is not known. The maximal infusion rate suggested by the manufacturer is $600 \mathrm{mg} / \mathrm{hr}$.

We found no evidence that vancomycin infusion over 1 hour in patients before cardiac operation is associated with any decrease in CO or SVR, contrasting with the animal studies of Cohen and associates. $^{3}$ As given in this study, anticipated plasma vancomycin levels are similar to the expected values in their dog/cat preparations at the time of peak myocardial depression. Perhaps the different outcomes in the whole animal preparations are related to different histamine levels, impurities in earlier preparations, ${ }^{15}$ or late manifestations of bolus injection, but these potential factors cannot explain why vancomycin caused myocardial depression in their isolated heart experiments.

In conclusion, vancomycin infusion of $1 \mathrm{gm}$ over 1 hour is safe and free of myocardial depression in patients before cardiac operation. The cardiac depression seen in animal studies was not observed in human beings. 


\section{REFERENCES}

1. Maki DG, Bohn JJ, Stolz SM, Kronche GM, Acher CW, Myerowitz PD. Comparative study of cefazolin, cefamandole, and vancomycin for surgical prophylaxis in cardiac and vascular operations: a double-blind randomized trial. J ThoraC CARdiovasc Surg 1992; 104:1423-34.

2. Fong IW, Baker CB, McKee DC. The value of prophylactic antibiotics in aorta-coronary bypass operations. J THORAC CARdIOVASC SuRg 1979;78:908-13.

3. Cohen LS, Wechsler AS, Mitchell JH, et al. Depression of cardiac function by streptomycin and other antimicrobial agents. Am J Cardiol 1970;26:505-11.

4. Valero R, Gomar C, Fita G. Adverse reactions to vancomycin prophylaxis in cardiac surgery. $\mathrm{J}$ Cardiothorac Vasc Anesth 1991;5:574-6.

5. Vonesh EF, Schork MA. Sample sizes in the multivariate analysis of repeated measurements. Biometrics 1986;42:601-10.

6. Southorn PA, Plevak DJ, Wright AJ, Wilson WR. Adverse effects of vancomycin administered in the perioperative period. Mayo Clin Proc 1986;61:721-4.

7. Frost E. Central nervous system trauma. Anesth Clin North Am 1987;5:565-85.

8. Caldwell TB. Infectious diseases. In: Katz J, Benumof $\mathrm{JL}$, Kadis LB, eds. Anesthesia and uncommon diseases. 3rd ed. Philadelphia: WB Saunders, 1990:703-5.
9. Newfield $P$, Roizen MF. Hazards of rapid infusion of vancomycin. Ann Intern Med 1979;91:581.

10. Rothenberg HJ. Anaphylactoid reaction to vancomycin. JAMA 1959;171:1101-2.

11. Romanelli VA, Howie MB, Myerowitz PD, et al. Intraoperative and postoperative effects of vancomycin administration in cardiac surgery patients: a prospective, double blind, randomized trial. Crit Care Med. 1993;21:1124-31.

12. Stier GR, McGory RW, Spotnitz WD, Schwenzer KJ. Hemodynamic effects of rapid vancomycin infusion in critically ill patients. Anesth Analg. 1990;71:394-399.

13. Wallace MR, Mascola JR, Oldfield EC. The red man syndrome: incidence, etiology and prophylaxis. 29th interscience conference on antimicrobial agents and chemotherapy 1989 Houston, Abstract 648.

14. Polk RE. Anaphylactoid reactions to glycopeptide antibiotics. J Antimicrob Chemother 1991;27(Suppl b):17-29.

15. Shenep JL, Hughes WT, Roberson PK, et al. Vancomycin, ticarcillin and amikacin compared with ticarcillin-clavulanate and amikacin in the empirical treatment of febrile neutropenic children with cancer. $\mathrm{N}$ Engl J Med 1988;319:1053-8. 